\title{
Although leukoplakia responds to some treatments relapses and adverse effects are common
}

\author{
What treatments are effective for treating oral leukoplakia?
}

\begin{abstract}
Lodi G, Sardella A, Bez C, Demarosi F, Carrassi A. Interventions for treating oral leukoplakia (Cochrane Review). In the Cochrane Library, Issue 3, 2004. Chichester, UK: John Wiley \& Sons.

Data sources The Cochrane Oral Health Group's Trials Register, Cochrane Central Trials Register, Medline and Embase were searched, as well as the following journals: Cancer, Community Dentistry and Oral Epidemiology, European Journal of Oral Sciences, Journal of Dental Research, Oral Oncology, Oral Surgery, Oral Medicine, Oral Pathology, Oral Radiology and Endodontics. The reference lists of included studies and reviews were checked, and oral medicine experts were contacted through a European mailing list (EURORALMED).

Study selection Randomised controlled trials (RCT) were included if they had enrolled patients who had a diagnosis of oral leukoplakia and who were undergoing any surgical or medical (topical and systemic) treatment. The primary outcome considered was malignant transformation of leukoplakia demonstrated by histopathological examination. Other outcomes considered were clinical resolution, histological modification and frequency of adverse effects.
\end{abstract}

Data extraction and synthesis Data were collected using a specific extraction form. The validity of studies included was assessed by two reviewers, on the basis of the method of allocation concealment, blindness of the study and loss of participants. Data were analysed by calculating relative risk (RR). When valid and relevant data were collected, a meta-analysis of the data was undertaken.

Results The possible effectiveness of surgical interventions, including laser therapy and cryotherapy, has never been studied by means of an RCT. Nineteen RCT of nonsurgical interventions were identified: seven were included. Vitamin A and retinoids were tested in five RCT (245 patients); the other drugs tested were bleomycin (one study), mixed tea (one study) and beta-carotene (one study). Malignant transformation was recorded in just two studies. None of the treatments tested showed a benefit compared with placebo. Treatment with betacarotene and vitamin A or retinoids was associated with significant rates of clinical resolution, compared with placebo or absence of treatment. Whenever reported, a high rate of relapse was a common finding. Side effects of variable severity were often described but interventions were well accepted by patients, since dropout rates were similar between treatment and control.

Conclusions To date there is no evidence of effective treatment in preventing malignant transformation of leukoplakia. Treatments may be effective in the resolution of lesions, but relapses and adverse effects are common.
Address for correspondence: Emma Tavender, Review Group Co-ordinator, Cochrane Oral Health Group, University Dental Hospital of Manchester, Higher Cambridge Street, Manchester M15 6FH, UK. E-mail: emma.tavender@man.ac.uk

\section{Commentary}

Oral leukoplakia is considered to be precancerous because about $4-6 \%$ of lesions turn into cancer. This incidence could be higher in lesions followed for a longer duration. ${ }^{1}$

Oral cancer causes substantial morbidity and mortality. The clinical form of the lesion is a poor indicator of the potential for such transformation. All leukoplakias, therefore, need to be assessed histopathologically, and treated vigorously if dysplasia is prominent.

This substantively amended Cochrane systematic review addresses the important issue of prevention of malignant transformation in oral leukoplakias. The review is well focused, with frequency of malignant transformation, clinical resolution, histopathological improvement, and safety and acceptability being the outcomes studied. The methods for identifying relevant trials and their quality assessment is robust. It is not clear from the review, however, whether data could be analysed separately for different clinical forms of leukoplakia. Other evidence ${ }^{2}$ suggests that proliferative verrucous leukoplakia has a very high risk for transformation to dysplasia and squamous cell carcinoma (The review did not include studies on proliferative verrucous leukoplakia as it is considered a separate entity, with different clinical, histology and prognostic features).

A small number of moderate-quality RCT, the small overall sample size $(n=386)$ and the short follow-up precluded the reviewers from providing a clinically useful bottom-line. Since its first publication in $2001,{ }^{3}$ only one study - which also had a medium risk of bias - could be added to this amended review. Currently, there is no RCT testing surgical treatment. It has been reported elsewhere that surgical treatment is no guarantee for preventing recurrence. ${ }^{4}$

The review is based upon seven RCT. The treatments studied include vitamin A, retinoids, bleomycin, beta-carotene and mixed tea. Of the seven, only two trials have a "low risk of bias". One of these $^{5}$ tested effectiveness of topical application of bleomycin, whereas the other ${ }^{6}$ investigated effectiveness of systemic 13-cisretinoic acid. Bleomycin clinically resolved lesions in 50\% of patients; systemic 13-cis-retinoic acid was not very effective in doing so. Nonetheless, both these agents were effective in preventing histological worsening with an RR of 0.53 [95\% confidence interval (CI), 0.23-1.22] and of 0.51 (95\% CI, $0.32-0.81$ ), respectively.

Systemic beta-carotene was most effective in providing clinical resolution, with an RR of 0.77 (95\% CI, 0.65-0.92). This trial, ${ }^{7}$ however, did not assess the lesions histopathologically. Two trials ${ }^{5,7}$ studied effectiveness in preventing malignant transformation. When compared with placebo, bleomycin, systemic beta-carotene and systemic vitamin A did not show any benefit.

Topical bleomycin, systemic 13-cis-retinoic acid, vitamin A (300,000 IU per week) and beta-carotene caused adverse effects of varying severity. These effects did not generally cause significant patient drop-out, however. 


\section{Practice points}

- All leukoplakias need to be assessed histopathologically for dysplasia.

- Systemic beta-carotene may provide significant clinical resolution.

- No treatment prevents malignant transformation, histopathological worsening or recurrence.

- Irrespective of response to therapy, long-term follow-up is essential.

\section{Shailesh Lele}

Bharati Vidyapeeth Deemed University Dental College and Hospital, Pune, India

1. Waldron CA, Shafer WG. Leukoplakia revisited. A clinicopathologic study of 3256 oral leukoplakias. Cancer 1975; 36:1386-1392.
2. Silverman Jr S, Gorsky M. Proliferative verrucous leukoplakia: a follow-up study of 54 cases. Oral Surg Oral Med Oral Pathol Oral Radiol Endod 1997; 84: 154-157.

3. Lodi G, Sardella A, Bez C, Demarosi F, Carrassi A. Interventions for treating oral leukoplakia (Cochrane Review). In the Cochrane Library, Issue 4. Oxford: Update Software; 2001:CD001829.

4. Schepman KP, van der Meij EH, Smeele LE:et al. Malignant transformation of oral leukoplakia: a follow-up study of a hospital based population of 166 patients with oral leukoplakia from the Netherlands. Oral Oncol 1998; 34:270-275.

5. Epstein JB, Wong FL, Millner A, Le ND. Topical bleomycin treatment of oral leukoplakia: a randomized double-blind clinical trial. Head Neck 1994; 16:539-544.

6. Hong WK, Endicott I, Itri LM, et al. 13-cis-retinoic acid in the treatment of oral leukoplakia. N Engl J Med 1986; 315:1501-1505.

7. Sankaranarayanan R, Mathew B, Varghese C, et al. Chemoprevention of oral leukoplakia with vitamin A and beta carotene: an assessment. Oral Oncol 1997 33:231-236.

Evidence-Based Dentistry (2005) 6, 15-16.

doi:10.1038/sj.ebd.6400311 